WESTFÄLISCHE WILHELMS-UNIVERSITÄT MÜNSTER

VOLKSWIRTSCHAFTLICHE DISKUSSIONSBEITRÄGE

\author{
Beitrag Nr. 371 \\ Vorläufige Fassung \\ Endfassung wird veröffentlicht im Jahrbuch für Recht und Ökonomik \\ des Dritten Sektors \\ (Hrsg. H.J. Schmidt-Trenz und R. Stober)
}

\title{
Verbände zwischen Markt und Staat aus \\ finanzwissenschaftlicher Sicht
}

\author{
von \\ Heinz Grossekettler \\ Münster 2006 \\ Institut für Finanzwissenschaft \\ D-48143 Münster, Wilmergasse $6-8$
}


Verbände zwischen Markt und Staat aus finanzwissenschaftlicher Sicht

von

Heinz Grossekettler

1. Problemstellung und Überblick

2. Exklusionsverbände 5

2.1 Struktur und Extensionsniveau von Exklusionsverbänden 5

2.2 Beispiele für legitime Exklusionsverbände im „Dritten Sektor“ 12

3. Engagementverbände 16

3.1 Struktur und Extensionsniveau von Engagementverbänden 16

3.2 Beispiele für legitime Engagementverbände im „Dritten Sektor“ 20

4. Fazit und Ausblick 24

5. Literatur 


\section{Problemstellung und Überblick}

Der „Dritte Sektor“ wird höchst unterschiedlich, zunehmend aber (auch) rechtlich abgegrenzt. Er umfasst in dieser Sicht Verbände, deren Basis das Vereinsrecht und die Spezialgesetzgebungen für öffentlich-rechtliche Institutionen bilden (so z.B. Schmidt-Trenz 2000, S. 14). Folgt man, wie es hier geschehen soll, dieser rechtlichen Abgrenzung, sind reine Marktprozesse ebenso aus der Betrachtung ausgeschlossen wie die Tätigkeiten der unmittelbaren Verwaltung der Gebietskörperschaften. Untersucht werden somit Verbände ,zwischen“ Markt und Staat.

Durchmustert man die Literatur zum so definierten „Dritten Sektor“, fällt auf, dass kaum thematisiert wird, welche Aufgaben die Verbände in diesem Sektor aus ordnungspolitischer Sicht verrichten und wie sie organisiert sein sollten. Es wird allenfalls gesagt, dass sie irgendwie Marktversagen und/oder Staatsversagen ausgleichen sollen; es wird aber nicht ausgeführt, warum dies erforderlich ist und wie der Ausgleich erfolgen soll. Außerdem findet man in der überwiegend soziologischen und politologischen Literatur keine Strukturierung des Sektors vor, die in ökonomisch überzeugender Weise mit den zu verrichtenden Aufgaben verknüpft ist.

Diese Forschungslücke füllen zu helfen, ist das Ziel dieses Beitrags. Es soll gezeigt werden, dass man den „Dritten Sektor“ mit Hilfe von wenigen Klassifikationsfragen so strukturieren kann, dass deutlich wird, welche Aufgaben die dort beheimateten Verbände aus ordnungsökonomischer Sicht übernehmen sollten und welche Verhaltensmaximen und Rechtsformen angemessen erscheinen.

Ausgangspunkt sollen dabei die folgenden vier Sachverhalte sein:

(1) Wie an Beispielen noch verdeutlicht werden wird, ist es für viele Verbände im „Dritten Sektor“ typisch, dass sie der Bereitstellung von verschiedenen Arten von Kollektivgütern dienen und nicht gewinnorientiert, sondern nach dem Bedarfsdeckungsprinzip arbeiten.

(2) Ausgehend von der Klassifizierungsfrage, ob die Kollektivgüterbereitstellung der Selbsthilfe oder der Fremdhilfe dient, kann man alle Verbände im „Dritten Sektor" in zwei Untergruppen unterteilen, die sich auch dadurch unterscheiden, wie sie ihre Finanzierung sicherstellen. Dies sind

- $\quad$ einerseits Exklusionsverbände, die der Selbsthilfe dienen und dabei auf den Ausschluss Beitragsunwilliger von der Nutzung ihres Verbandsgutes setzen, und 
- andererseits Engagementverbände, die der Fremdhilfe dienen und auf freiwillige Beiträge und/oder Staatszuschüsse vertrauen. ${ }^{1}$

Es ist allerdings möglich, Verbände beider Arten unter einem gemeinsamen organisatorischen Dach zusammenzufassen, und dies spielt - man denke etwa an Organisationen der Wohlfahrtspflege wie das Deutsche Rote Kreuz (Hüdepohl 1996) - in der Realität eine nicht unerhebliche Rolle.

(3) Von der vorstehenden Einteilung nicht erfasst werden politische Interessenverbände, die normalerweise allerdings auch nicht zum „Dritten Sektor“ gerechnet werden. Sie zeichnen sich dadurch aus, dass sie wie Exklusionsverbände Selbsthilfezwecken dienen sollen, dass ihnen aber wie Engagementverbänden eine privatrechtliche Exklusionsmöglichkeit fehlt und eine öffentlich-rechtliche in der Regel verweigert wird.

(4) Je nach den Eigenarten des Kollektivgutes, das die Verbände jeweils bereitstellen und manchmal auch herstellen müssen, benötigen sie den Zugriff auf unterschiedliche Finanzierungsmittel. Hieraus folgt, dass man die Verbände im „Dritten Sektor" aus ökonomischer Sicht danach klassifizieren kann, welche Organisations- und Finanzierungsform mit Blick auf das jeweilige Verbandsgut ordnungspolitisch adäquat erscheint.

Verbände, die eine aus ordnungspolitischer Sicht angemessene Aufgabe erfüllen sowie die dazu passende Organisations- und Finanzierungsform aufweisen, werden im Folgenden als „legitime Verbände“ bezeichnet. Es gibt im „Dritten Sektor“ allerdings auch Verbandsstrukturen, die ordnungspolitisch unangemessen erscheinen; sie werden in diesem Aufsatz nicht betrachtet. ${ }^{2}$ Mittels der Beschränkung auf legitime Verbände soll gezeigt werden, dass es einen Bedarf für solche Verbände gibt und dass die Existenz des legitimen Teils des „Dritten Sektors“ nicht etwa auf irgendeine Form von Staatsversagen zurückzuführen ist, sondern dass Staatsversagen vielmehr dann vorläge, wenn die Erledigung legitimer Verbandsaufgaben in die unmittelbare Staatsverwaltung integriert würde. Zunächst sollen Strukturen und Erscheinungsformen legitimer Exklusionsverbände erläutert werden, anschließend solche legitimer Engagementverbände.

1 Um Missverständnissen vorzubeugen, sei ausdrücklich darauf hingewiesen, dass die Unterscheidung von Exklusions- und Engagementverbänden auf einer anderen Ebene liegt als die von ,exklusiven“ und „,inklusiven“ Gruppen, die Olson (1968, S. 35-41) in die Literatur eingeführt hat. Ähnlichkeit besteht dagegen mit Putnams (2001, S. 27 f.) Unterscheidung innenund außenorientierten Sozialkapitals.

2 Beschränkt man sich nicht auf legitime Verbände, fällt es schwer, eine Ordnung in die Verbands- und Finanzierungsstrukturen zu bringen, die im „Dritten Sektor“ vorfindbar sind. Zu einer rein positiven Klassifikationen der Totalität der Verbände in diesem Sektor vgl. vor allem Gelbhaar (1998), Lepelmeier (1984) und Zimmermann/Krenzer (2001); eine Übersicht über die Spezifika der Finanzierungsformen bietet Haibach (1998). Eine Bestandsaufnahme von Tätigkeitsbereichen und eine wohlfahrtsökonomische Analyse, die sich stark auf Markt- und Kontraktversagen stützt und Entwicklungshemmnisse für Non-Profit-Organisationen in Deutschland herausarbeitet, findet man bei Kraus/Stegarescu (2005). 


\section{Exklusionsverbände}

\subsection{Struktur und Extensionsniveau von Exklusionsverbänden}

Die Struktur von Exklusionsverbänden ist im Kasten 1 in idealisierter Form dargestellt. Man kennt sie z.B. aus der üblichen Organisation von Sportvereinen: Es gibt ein mehr oder weniger stark ausdifferenziertes Verbandsmanagement (den Vorstand), das den Verband nach außen repräsentiert. Es sorgt dafür, dass die Verbandsmitglieder das Verbandsgut nutzen können und dass auch die notwendigen Vorleistungen beschafft werden. Zwischen dem Management und den Mitgliedern besteht keine Markt-, sondern eine Mitgliedschaftsbeziehung, die Nutzungs- und Kontrollrechte umfasst. Zwischen dem Management und den Vorleistungslieferanten besteht dagegen im Normalfall eine Marktbeziehung; nur in bestimmten Ausnahmefällen ist es gemäß den öknomischen „Make or buy“-Kriterien angemessen, das Kaufen von Vorleistungen durch ein Selbermachen zu ersetzen.

Generell zeichnen sich Exklusionsverbände durch die folgenden drei Eigenschaften aus:

- $\quad$ Sie stellen ein Kollektivgut bereit, dessen Nutzung ausschließlich der Wohlfahrt der eigenen Mitglieder dienen soll. Bei der Nutzung des Gutes entsteht im Normalfall keine Rivalität.

- Zur Finanzierung der Kollektivgüterbereitstellung wird das Exklusionsprinzip eingesetzt. Es kommt also nur derjenige in den Genuss der Verbandsgutes, der als Mitglied auch einen Beitrag zur Finanzierung leistet. In dieser Hinsicht gibt es somit eine Analogie zur Versorgung mit einem Individualgut: Nur wer über eine hinreichende Zahlungsbereitschaft verfügt, kann das Gut nutzen.

- Der Verband soll aus der Sicht seiner Mitglieder keine Gewinne machen, sondern dem Bedarfsdeckungsprinzip folgen und sich über Entgeltzahlungen der Mitglieder finanzieren; die Mitglieder drängen darauf, dass der Barwert dieser Zahlungen möglichst niedrig ist, und überwachen das Management in dieser Hinsicht. 


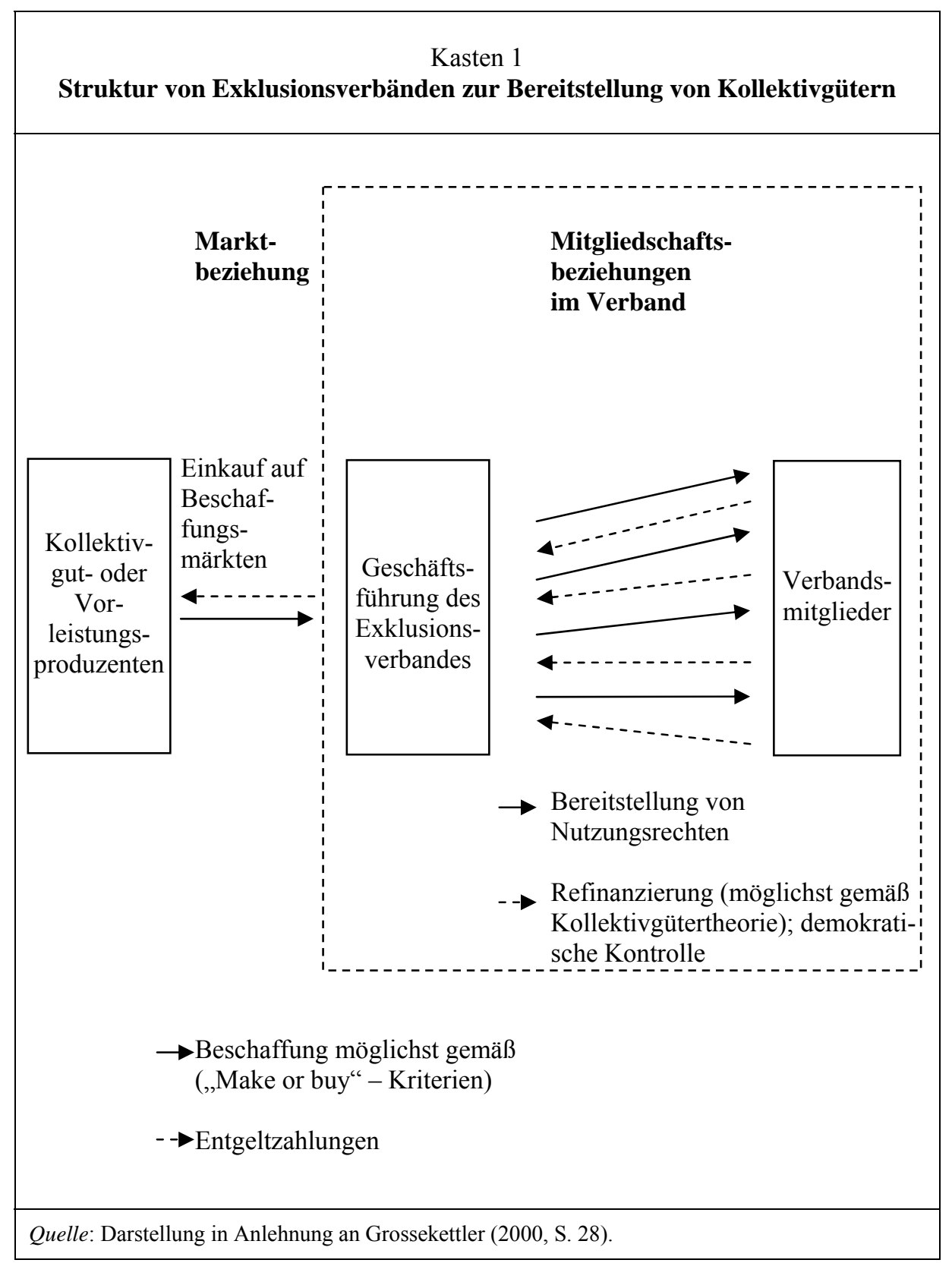

Kombiniert man das Interesse der Mitglieder mit der volkswirtschaftlichen Forderung nach Vermeidung externer Kosten, ergibt sich die ordnungspolitische Forderung, dass Exklusionsverbände nach dem Kongruenzprinzip organisiert sein sollten. 
Dieses wird im Kasten 2 ausführlich erläutert. Es fordert im Wesentlichen eine gruppenmäßige Äquivalenz von Leistung und Gegenleistung und das Vorhandensein von Anreizen für das Verbandsmanagement, umfassende und sorgfältige NutzenKosten-Abwägungen zu treffen und vor den Mitgliedern dafür gerade zu stehen.

Zur Durchsetzung des Kongruenz- und speziell auch des darin enthaltenen Exklusionsprinzips stehen unterschiedliche rechtliche Möglichkeiten zur Verfügung. Sie ergeben sich aus einer Skala von Verbandsorganisationsmöglichkeiten, die im Kasten 3 erläutert wird. Diese Skala beschreibt in den mittleren beiden Spalten das so genannte Extensionsniveau der Finanzierungskompetenz eines Verbandes und ist vom Skalentyp her eine so genannte Sukzessivkategorienskala. Dies bedeutet, dass Klassen von Verbänden eines bestimmten Typs in eine Rangfolge gebracht werden, dass es zwischen den Elementen innerhalb ein und derselben Verbandsklasse aber keine Rangordnung gibt.

Die Rangordnung im Kasten 3 ergibt sich mit Blick auf zwei miteinander verbundene Sachverhalte. Diese sind einerseits die jeweils mit einem bestimmten Exklusionsmodus gekoppelte Finanzierungskompetenz - die Menge und Art der Finanzierungsmöglichkeiten - und andererseits der Verdünnungsgrad von Eigentumsrechten: Ein jeweils höherer Verbandstyp verfügt über mehr Finanzierungsmöglichkeiten als ein niedrigerer, und die Eigentumsrechte der Mitglieder sind im höheren Verband typischerweise stärker verdünnt.

Die beiden Kategorien von Exklusionsverbänden, die für den „Dritten Sektor“ von Bedeutung sind, sind im Kasten durch einen hellen Hintergrund gekennzeichnet. Es sind Privatvereine mit dem Extensionsniveau 1 und Zwangsvereine mit dem Extensionsniveau 2 .

Privatvereine wie z.B. ein Tennisklub haben finanzierungstechnisch die Kompetenz, Preise von Nichtmitgliedern und Entgelte in Form von Gebühren und Beiträgen von den Mitgliedern zu erheben. Zahlungsunwillige können sie auf der Basis des Privatrechts von der Nutzung ihres Verbandsgutes ausschließen. Die Gebühren sollten aus ökonomischer Sicht der Abdeckung von Grenzkosten, die Beiträge der Abdeckung von Fixkosten dienen (Grossekettler 2003, S. 584). Diese Sichtweise ist eine andere als die vieler Vereinsmanager in der Praxis. Deren Denken ist vom herrschenden Entgeltrecht geprägt. Deshalb werden Gebühren vielfach auch dann erhoben, wenn Leistungen zwar individuell zurechenbar sind, aber - wie normalerweise z.B. bei Museumsbesuchen - gar keine Grenzkosten anfallen; auf der anderen Seite wird - vermutlich aus politischen Gründen - auf eine Gebührenerhebung verzichtet, 
obwohl - wie z.B. bei der Pkw-Nutzung von Autobahnen in Hochlastzeiten Grenzkosten anfallen. ${ }^{3}$

\section{Kasten 2 \\ DAS KONGRUENZPRINZIP}

Das Kongruenzprinzip zielt darauf ab, die Versorgung mit einem Gut so zu gestalten, dass Entscheidungsbefugnis, Nutzen und Kosten in einer Weise zusammen fallen, die der Situation bei den meisten Individualgütern möglichst nahe kommt: Wer Brot kauft, fällt selbst die Entscheidung dafür (Selbstverwaltung), hat den Nutzen davon (Selbsthilfe, d.h. Förderung der eigenen Wohlfahrt) und muss die Kosten tragen (Selbstverantwortung). ${ }^{*}$ Im Sinne dieser Zielsetzung verlangt das Kongruenzprinzip zweierlei:

* Der Kreis der Nutzenempfänger soll sich mit dem Kreis der Kostenträger decken, d.h.

- niemand soll Nutzen empfangen, ohne an den Kosten beteiligt zu sein (d.h. Exklusion von Zahlungsunwilligen), und

- niemand soll Kosten tragen müssen, ohne am Nutzen zu partizipieren (d.h. Vermeidung externer Kosten).

Diese Forderung nach ausschließlicher Selbsthilfe und vollständiger Selbstverantwortung nennt man Prinzip der fiskalischen Äquivalenz.

* Der Kreis derjenigen, die Versorgungsentscheidungen unterworfen sind, soll das Recht haben, über Wahlen direkt diejenigen kontrollieren zu können, die Versorgungsentscheidungen fällen, und zwar so, dass die Entscheider uno actu für den Nutzen und die Kosten verantwortlich gemacht (und so zu einer wohlabgewogenen Entscheidung gedrängt) werden, dass also nicht bei einer Wahl 1 nur der Nutzen eine Rolle spielt und bei einer Wahl 2 nur die Kosten relevant sind.

Diese Forderung nach Selbstverwaltung heißt Prinzip der ungeteilten demokratischen Nutzen-Kosten-Verantwortung.

* Die Klammerzusätze verweisen auf die Raiffeisengrundsätze, in denen das Kongruenzprinzip der Sache (nicht aber dem Namen) nach erstmalig formuliert wurde (vgl. hierzu Mändle 1992).

Quelle: eigene Darstellung.

3 Dass die vom Autor eingeführte Regel „Finanziere die bei der Nutzung mancher Kollektivgüterarten auftretenden Grenzkosten über Gebühren und die Restkosten über (Zwangs-) Beiträge" effizienzfördernd ist, wird in der ökonomischen Literatur nicht bestritten. Es werden aber z.T. modifizierte Regeln empfohlen, so z.B. von Märkt (2003), der die Einbeziehung solcher Regeln in eine anreizkompatible Steuerordnung fordert. 


\begin{tabular}{|c|c|c|c|}
\hline \multicolumn{4}{|c|}{$\begin{array}{c}\text { Kasten } 3 \\
\text { Das Extensionsniveau e von Exklusionsverbänden }\end{array}$} \\
\hline $\begin{array}{c}\text { E-WERT } \\
\text { (SUKZESSIV- } \\
\text { KATEGORIEN- } \\
\text { SKALA) } \\
\end{array}$ & BEZEICHNUNG & $\begin{array}{l}\text { FINANZIERUNGSKOMPETENZ } \\
\text { UND EXKLUSIONSTECHNIK }\end{array}$ & $\begin{array}{l}\text { TYPISCHER VER- } \\
\text { WÄSSERUNGS- } \\
\text { GRAD DER EIGEN- } \\
\text { TUMSRECHTE }\end{array}$ \\
\hline 0 & $\begin{array}{l}\text { Individualniveau } \\
\text { (unechter Ver- } \\
\text { band) }\end{array}$ & $\begin{array}{l}\text { Kalkulation von Schatten- } \\
\text { preisen } \\
\text { (privatrechtliche Exklusion) }\end{array}$ & 0 \\
\hline 1 & $\begin{array}{l}\text { Privatvereins- } \\
\text { niveau } \\
\text { (z.B. Tennisklub) }\end{array}$ & $\begin{array}{l}\text { Nutzungspreise, Gebühren } \\
\text { und freiwillige Beiträge } \\
\text { (privatrechtliche Exklusion) }\end{array}$ & $0<\mathrm{e}<2$ \\
\hline 2 & $\begin{array}{l}\text { Zwangsvereins- } \\
\text { niveau } \\
\text { (z.B. Deichver- } \\
\text { band) }\end{array}$ & $\begin{array}{l}\text { wie bei e = } 1 \text { plus Zwangs- } \\
\text { beiträge } \\
\text { (öffentlich-rechtliche Exklu- } \\
\text { sion) }\end{array}$ & $2<\mathrm{e}<3$ \\
\hline 3 & Kommunalniveau & $\begin{array}{l}\text { wie bei e }=2 \text { plus lokale } \\
\text { Steuern } \\
\text { (öffentlich-rechtliche Exklu- } \\
\text { sion) }\end{array}$ & $3<\mathrm{e}<4$ \\
\hline 4 & Landesniveau & $\begin{array}{l}\text { wie bei e }=3 \text { plus regionale } \\
\text { Steuern } \\
\text { (öffentlich-rechtliche Exklu- } \\
\text { sion) }\end{array}$ & $4<\mathrm{e}<5$ \\
\hline 5 & Bundesniveau & $\begin{array}{l}\text { wie bei e }=4 \text { plus nationale } \\
\text { Steuern } \\
\text { (öffentlich-rechtliche Exklu- } \\
\text { sion) }\end{array}$ & $5<e<6$ \\
\hline 6 & $\begin{array}{l}\text { Wirtschafts- } \\
\text { gemeinschafts- } \\
\text { niveau }\end{array}$ & $\begin{array}{l}\text { Fortsetzung der Skala denk- } \\
\text { bar, in der Realität aber noch } \\
\text { nicht beobachtbar; stattdessen }\end{array}$ & $6<\mathrm{e}<7$ \\
\hline 7 & Weltniveau & nationale Beiträge & $7 \leq \mathrm{e}$ \\
\hline
\end{tabular}


Die zweite im Kasten 3 gezeigte Variable ist der Verdünnungsgrad der individuellen Eigentumsrechte der Mitglieder am Verbandsgut. Er ist umso niedriger, je höher die Chance eines typischen Mitglieds ist, seine eigenen Vorstellungen über die Nutzung des Verbandsguts durchsetzen zu können. Diese Chance hat bei Alleineigentümern den Wert Eins. Sie fällt mit steigender Zahl und Heterogenität der Verbandsmitglieder, die - wirtschaftlich gesehen - ja Miteigentümer sind und deren Anzahl und Heterogenität für steigende Konsensfindungskosten verantwortlich sind. Bei Privatvereinen hat der Verdünnungsgrad der Eigentumsrechte einen relativ geringen Wert, weil die Chancen zur Durchsetzung von Vorstellungen über den Umgang mit dem Verbandsgut aufgrund der typischen Interessenhomogenität der Mitglieder relativ hoch und die Konsensfindungskosten deshalb niedrig sind.

Zwangsvereine wie z.B. ein Deichverband können über die gleichen Finanzierungsmittel verfügen wie Privatvereine, darüber hinaus aber auch noch Zwangsbeiträge erheben. Zahlungsunwillige werden dadurch von der Nutzung ausgeschlossen, dass z.B. das Halten eines Grundstücks in einem von einem Deich geschützten Raum oder das Ausüben bestimmter Berufstätigkeiten nur denjenigen gestattet wird, die auch Mitglied im Zwangsverein sind. Anders formuliert besteht hier auf der Grundlage von öffentlich-rechtlichen Spezialgesetzen ein Beitritts- und ein Beitragszwang. Wer sich dem entziehen will, muss das Grundstück hinter dem Deich oder die bestimmte Art von Berufstätigkeit aufgeben, wird also von den Mitgliedsrechten auf öffentlich-rechtliche Weise exkludiert. Das alte preußische Deichrecht hat dies früher sehr plastisch ausgedrückt: „Wer nicht will deichen, der muss weichen." Wegen der meist stärkeren Heterogenität der Mitgliederinteressen und der daraus resultierenden Konflikte sind die Konsensfindungskosten und damit auch der Verdünnungsgrad der Eigentumsrechte bei den Zwangsvereinen typischerweise gröBer als in Privatvereinen.

Der Unterschied zwischen dem hellen und dem dunklem Bereich im Kasten 3 wird deutlich, wenn man einmal die Verbandstypen betrachtet, die an den hellen Bereich angrenzen.

Individuen, deren Extensionsniveau der Finanzierungskompetenz unterhalb des markierten Bereichs der Vereine und Zwangsvereine liegt, können im Rahmen ihrer Eigenversorgung von sich selbst als Quasimitglied in einem unechten Verband natürlich überhaupt keine Entgelte im eigentlichen Sinn erheben. Sie können lediglich mit Opportunitätskosten rechnen, d.h. mit Schattenpreisen. Das Extensionsniveau ihrer Finanzierungskompetenz hat deshalb den Wert Null. Die Quasiverbandsgüter von Individuen sind Individualgüter; andere Individuen können auf der Basis des Privatrechts von der Nutzung ausgeschlossen werden. Der Grad der Verdünnung der Eigentumsrechte hat den Wert Null, weil die Chance zur Durchsetzung eigener Vorstellungen bei individuellem Privateigentum den Wert Eins hat. 
Kommunen, deren Extensionsniveau oberhalb des Bereichs der Vereine und Zwangsvereine liegt, unterscheiden sich von Zwangsvereinen dadurch, dass sie nicht auf ganz bestimmte Tätigkeiten festgelegt sind, sondern in bestimmten Grenzen über eine Kompetenzkompetenz verfügen. Ein Teil ihrer Einnahmen (die Kommunalsteuern) unterliegt deshalb auch keiner indirekten (ökonomischen) Zweckbindung für das Vorhalten ganz bestimmter Verbandseinrichtungen. Neben ihren - ex definitione nicht zweckgebundenen - Steuern nutzen sie aber natürlich auch die Finanzierungsmittel der Privat- und der Zwangsvereine, also etwa Gebühren (z.B. für den Besuch von kommunalen Museen), freiwillige Beiträge (z.B. für den Besuch von Kindergärten) oder Zwangsbeiträge (z.B. als Anliegerbeiträge). Exkludieren können Kommunen sowohl privatrechtlich als auch öffentlich-rechtlich. Die Eigentumsrechte sind bei ihnen aus der Sicht der sehr heterogenen individuellen Mitglieder noch stärker verdünnt als bei Zwangsvereinen.

Bisher wurde die ordnungspolitische Forderung erhoben, dass Exklusionsverbände im Interesse der Mitglieder und der Volkswirtschaft nach dem Kongruenzprinzip organisiert sein sollten. Außerdem wurden verschiedene Möglichkeiten aufgezeigt, das Kongruenz- und damit gleichzeitig auch das Exklusionsprinzip zu realisieren. Dabei zeigte sich, dass ein steigendes Extensionsniveau aus der Sicht eines einzelnen Mitglieds mit steigenden Konsensfindungskosten und einer Verdünnung von Eigentumsrechten einhergeht. Eine Verdünnung von Eigentumsrechten stellt ökonomisch ein Übel dar, dessen Ausmaß möglichst niedrig gehalten werden sollte. Hieraus ergibt sich eine bisher noch nicht diskutierte ordnungspolitische Forderung an Exklusionsverbände: Die Ausstattung von Exklusionsverbänden mit Finanzierungskompetenz sollte dem Subsidiaritätsprinzip genügen, um eine unnötige Verdünnung von Eigentumsrechten und damit einhergehende Unwirtschaftlichkeiten zu vermeiden. Wenn ein Kollektivgut von einem Privatverein bereitgestellt werden kann, sollte man es also nur beim Vorliegen besonderer Gründe von einem Zwangsverein oder gar von einer Kommune oder einem noch höheren Verband bereitstellen lassen. Dies bedeutet, dass stets nur derjenige Verbandstyp als legitimer Aufgabenträger anzusehen ist, der möglichst „,bürgernah“ ist und gerade noch über dasjenige Extensionsniveau verfügt, das zur Realisation der richtigen Finanzierung und des Kongruenzprinzips erforderlich ist. Geht man von einem vorgegebenen Kollektivgut aus, muss man bei der Suche nach einem legitimen Verband als Aufgabenträger aus ökonomischer Sicht somit folgende Fragen stellen:

- Wie sollte das Gut finanziert werden: über einen gemischten Tarif aus Grenzkostengebühren und freiwilligen bzw. Zwangsbeiträgen, über einen reinen (Zwangs-) Beitragstarif oder über lokale, regionale oder nationale Steuern?

- Welche Verbandstypen könnten sowohl die richtige Finanzierung als auch das Kongruenzprinzip realisieren?

- Falls es - was selten der Fall sein dürfte - mehrere solcher Verbandstypen gibt: Welcher von ihnen würde dem Subsidiaritätsprinzip genügen, d.h. das Kongruenzprinzip auf einem möglichst geringen Extensionsniveau realisieren? 
Mit Hilfe dieser Fragen kann man einerseits vorgegebenen Gütern ordnungspolitisch legitime Verbandstypen zuordnen und andererseits prüfen, ob ein konkreter Verband im Hinblick auf die von ihm tatsächlich bereit gestellten Güter als legitimer Versorgungsverband betrachtet werden kann. Die zuerst genannte Klassifikationsmöglichkeit - die Zuordnung von Gütern zu legitimen Verbandstypen - entspricht ordnungspolitischen Organisationsempfehlungen und soll im nun folgenden Abschnitt an Beispielen erläutert werden.

\subsection{Beispiele für legitime Exklusionsverbände im „Dritten Sektor“}

In diesem Abschnitt werden zwei Beispiele für legitime Exklusionsverbände auf dem Vereinsniveau betrachtet - ein Tennisverein (1) und eine lokale Elektrizitätsversorgungsgenossenschaft (2) - sowie vier Beispiele für Verbände auf dem Zwangsvereinsniveau: ein Deichverband (3), eine IHK (4), ein Business Improvement District (5) und eine Abwassergenossenschaft (6). Die Güter dieser Verbände könnten jeweils auch in anderen Organisationsformen - auf einem höheren Extensionsniveau - bereitgestellt werden; dies würde aber jedenfalls dann gegen ökonomische Prinzipien wie das Exklusionsprinzip, das Kongruenzprinzip und das Subsidiaritätsprinzip verstoßen und Unwirtschaftlichkeiten zur Folge haben, wenn es keine besonderen Rechtfertigungsgründe wie etwa Verwaltungsverbundvorteile oder eine bessere Beherrschung von Machtstrukturen gäbe.

(1) Tennisverein

Aufgrund der Kapazität an Verfügbarkeitsstunden, die ein einzelnes Spielerpaar normalerweise nicht auslasten kann, ist eine Tennisanlage als ein Kollektivgut einzustufen, das bei allen Mitgliedern im Bereitstellungsverband zu Kosteneinsparungen führt. So lange keine Überfüllungserscheinungen auftreten, ist eine Anlage praktisch grenzkostenfrei nutzbar und sollte deshalb beitragsfinanziert werden. Zur Abwehr von Überfüllung in Hochlastzeiten sollten Gebühren erhoben (oder ein anderes Rationierungsverfahren angewandt) werden. Da privatrechtlich exkludiert werden kann, wäre die erforderliche Finanzierungskompetenz auf dem Privatvereinsniveau gegeben. Hier könnte auch das Kongruenzprinzip realisiert werden. Nach dem Subsidiaritätsprinzip ist eine Tennisanlage folglich ihrer ökonomischen Natur nach ein Klubkollektivgut. Der zugehörige Tennisverein gehörte zum „Dritten Sektor“ und würde für eine optimale Bereitstellung sorgen. Würde eine Tennisanlage dagegen z.B. von einer Gemeinde bereitgestellt - was es in der Realität gibt -, würden sowohl das Kongruenz- als auch das Subsidiaritätsprinzip verletzt und käme es deshalb zu Unwirtschaftlichkeiten: Nichtmitglieder würden zur Finanzierung herangezogen, und/oder die Finanzierung erfolgte über Durchschnittskosten-Gebühren, die zu falschen Verdrängungseffekten führen würden, und/oder nicht Betroffene wären mitspracheberechtigt. 
(2) Lokale Elektrizitätsversorgungsgenossenschaft

In ein lokales Stromverteilungsnetz wird Strom von Erzeugern und Handelsgesellschaften eingespeist und an die örtlichen Verbraucher weitergeleitet. Es muss ein Unternehmen geben, das als Träger des Netzes fungiert. Beim Verbrauch von Strom fallen Grenzkosten an, die jedoch nicht so hoch sind, dass ein Grenzkostenpreis die Durchschnittskosten der Netzvorhaltung mit abdecken würde. Deshalb muss ein zweiteiliger Tarif in Form einer Grenzkostengebühr und eines Bereitstellungsbeitrages erhoben werden. Da privatrechtlich exkludiert werden kann, würde ein Privatverein aller lokalen Stromverbraucher sowohl über die notwendige Finanzierungskompetenz verfügen als auch dem Subsidiaritätsprinzip und dem Kongruenzprinzip genügen. Da der lokale Netzbetreiber dem Nachfragerverein gegenüber aber die Stellung eines natürlichen Monopols inne hätte und damit auch über Ausbeutungsmöglichkeiten verfügen würde, wäre es sinnvoll, zu einer vertikalen Integration zwischen Verein und Netzbetreiber zu greifen. Dies würde wohlfahrtsschädliche Monopolaufschläge verhindern und einen Anreiz zum Streben nach niedrigen Grenz- und Durchschnittskosten setzen. Eine zweckmäßige Rechtskonstruktion für solch einen Verein mit integriertem Unternehmen ist das Genossenschaftsrecht (Grossekettler 1984). Es läge also ein Genossenschaftsgut vor. Im vorliegenden Fall hätte die Elektrizitätsversorgungsgenossenschaft die Aufgabe, die Vereinsmitglieder über Grenzkostengebühren und Fixkostenbeiträge unter Verzicht auf eine Monopolrente mit Strom zu versorgen. Sie verfügte über alle Anreize für eine gute Versorgungsleistung und gehörte zum „Dritten Sektor“. Von der Normalstruktur im Kasten 3 unterschiede sie sich aber durch die Integration des Leistungsproduzenten. Diese Integration genügte den ökonomischen „Make or buy“-Kriterien, denn sie würde die Ausbeutungsgefahr transaktionskostengünstig abwenden und damit die Summe aus Transformations- und Transaktionskosten minimieren. Elektrizitätsversorgungsgenossenschaften gab es früher häufiger. Seit der Kommunalisierung der Stromversorgung spielten sie aber lange Zeit keine Rolle mehr. Die heutige Versorgungsstruktur über Erwerbsunternehmen und kommunale Stadtwerke liegt außerhalb des „,Dritten Sektors"; sie ist der vorgestellten Genossenschaftslösung angesichts des fehlenden Wettbewerbs in der Elektrizitätsversorgung und des Hintereinanderschaltens von mehreren Monopolen mit ihren Aufschlägen auf die Grenzkosten aber unterlegen. ${ }^{4}$ Vermutlich ist dies der Grund dafür, dass neuerdings wieder lokale Elektrizitätsversorgungsgenossenschaften gegründet werden, etwa von den Nutzern nachwachsender Rohstoffe zur Stromversorgung.

4 Die schlechteste Organisationsform, die es ökonomisch für Wertschöpfungsketten gibt, sind hintereinander geschaltete Monopole, bei denen die Monopolaufschläge stromaufwärts liegender Monopole in die Grenzkosten weiter stromabwärts liegender eingehen. Vertikale Integration kann in solch einem Fall, wie er z.B. in der deutschen Strom- und Gaswirtschaft vorliegt, dazu führen, dass sowohl die Konsumentenversorgung über niedrigere Preise und höhere Mengen verbessert wird als auch die Gewinnsituation der Kapitalgeber. 


\section{(3) Deichverband}

Aufgrund der Unabhängigkeit der Schutzleistung von der Zahl der Geschützten ist ein Deich ein lokales Kollektivgut, dessen Nutzung keine Grenzkosten verursacht. Also ist eine reine Beitragsfinanzierung angebracht, die z.B. nach Ertragswerten der geschützten Grundstücke differenziert werden könnte. Da nicht privatrechtlich exkludiert werden kann, muss zu einer öffentlich-rechtlichen Exklusion gegriffen werden. Die erforderliche Finanzierungskompetenz muss folglich Zwangsbeiträge umfassen. Nach dem Subsidiaritätsprinzip ist somit ein Zwangsverein als Bereitstellungsgarant zu wählen, der alle Deichhinterleger umfasst und das Kongruenzprinzip erfüllt. Solche Deichverbände findet man in Deutschland in Niedersachsen, nicht aber in Schleswig-Holstein. Sie gehören zum „Dritten Sektor“. Eine Bereitstellung durch die Verwaltung eines Bundeslandes (wie in Schleswig-Holstein) verletzt dagegen das Subsidiaritäts- und das Kongruenzprinzip.

\section{(4) IHK}

Die Kernleistung einer IHK besteht in der Interessenvertretung der Unternehmen einer Region gegenüber Staat und Kommunen. Daneben gibt es eine Fülle weiterer Leistungen, auf die hier nicht eingegangen wird. Bei der Erbringung der Kernleistung fallen bei der Erweiterung der Unternehmenszahl kaum Grenzkosten an. Also ist eine Beitragsfinanzierung angemessen. Da von der Interessenvertretung alle Unternehmen profitieren und eine privatrechtliche Exklusion nicht möglich ist, muss öffentlich-rechtlich exkludiert und ein differenzierbarer Zwangsbeitrag erhoben werden. Nach dem Subsidiaritätsprinzip liegt deshalb ein Zwangsvereinsgut vor. Ein regionaler Zwangsverein würde dem Kongruenzprinzip entsprechen und ist im heutigen Recht auch realisiert. Die Bereitstellung von IHK-Leistungen durch den „Dritten Sektor“ ist ökonomisch sinnvoll (differenzierter hierzu Schmidt-Trenz 1996).

\section{(5) Business Improvement District}

Business Improvement Districts tragen zur Entwicklung von Stadtteilen über die Bereitstellung von Leistungen wie vor allem den folgenden bei: Freundlichkeit des Erscheinungsbildes, Standortmarketing, Informationsstellen, Weihnachts- bzw. Festbeleuchtungen und die so genannte Möblierung des öffentlichen Raums (IHK Hamburg 2004, S. 20 f. und S. 44). Diese Leistungen kommen direkt vor allem den Gewerbetreibenden und Grundstücksbesitzern zugute, indirekt auch den Mietern. Die von den Leistungen des Districts verursachten Kosten sind von der Zahl der Profitierenden in der Regel unabhängig. Sie können jedoch - je nach den geplanten Aktionen - im Zeitablauf unterschiedlich hoch sein und unterschiedliche Teilgruppen im Stadtteil in unterschiedlich hoher Weise begünstigen. Deshalb wäre eine Finanzierung über - gegebenenfalls z.B. nach Ertragswertsteigerungen differenzierte Beiträge angemessen. Da privatrechtlich nicht exkludiert werden kann, ergibt sich nach dem Subsidiaritätsprinzip ein Zwangsverein als Organisationsform der Wahl. Eine solche Organisationsform würde auch dem Kongruenzprinzip genügen und wä- 
re im „Dritten Sektor“ angesiedelt, der damit auch hier wieder für eine angemessene Bereitstellung sorgen würde.

(6) Abwassergenossenschaft

Eine der ältesten deutschen Umweltschutzmaßnahmen war die 1904 erfolgte Gründung der Emscher-Genossenschaft. Ihre Leistungen bestehen in der Reinhaltung des Flusswassers über Abwasserreinigungsanlagen und Klärwerke, der Weiterverwertung von Klärgut und der Regulierung des Flusses über Pumpwerke. Begünstigt sind die Flussanlieger in einer großen Zahl von Stadt- und Landkreisen. Da privatrechtlich nicht exkludiert werden kann, mussten die äußerst heterogenen Mitglieder in einem funktionellen Zwangsverein zusammengefasst werden, was auch dem Kongruenzprinzip entspricht. Da selbstständige Reinigungs- und Regulierungsbetriebe außerdem jeweils über die Stellung eines natürlichen Monopols verfügt hätten, wurde der Verein richtigerweise mit den Produktionsbetrieben $\mathrm{zu}$ einer Zwangsgenossenschaft zusammengefasst. Auch diese stellt einen legitimen Bestandteil des „Dritten Sektors“ dar.

Insgesamt zeigen die besprochenen Beispiele, dass über die Anwendung der Kriterien „optimale Finanzierung“, „Kongruenzprinzip“ und „Subsidiaritätsprinzip“ von Exklusionsverbänden im „Dritten Sektor“ viele Leistungen wohlfahrtsoptimal bereitgestellt werden können. Die potenzielle soziale Rentabilität der Exklusionsverbände im „Dritten Sektor“ und ihre wohlfahrtsökonomische Legitimität ist damit nachgewiesen. Außerdem ist gezeigt worden, dass legitime Exklusionsverbände nicht etwa nur Hilfskonstruktionen zur Erbringung von Leistungen sind, die an sich Aufgaben der Staatsverwaltung gewesen wären, sondern dass sie - jedenfalls aus finanzwissenschaftlicher Sicht - eine eigenständige Existenzberechtigung haben. Würde die Staatsverwaltung (die unmittelbare Verwaltung der Gebietskörperschaften) typische Leistungen des „Dritten Sektors“ übernehmen, wie sie vorstehend an Beispielen erläutert wurden, müsste diese Missachtung ordnungspolitischer Prinzipien als ,Staatsversagen“" klassifiziert werden. 


\section{Engagementverbände}

\subsection{Struktur und Extensionsniveau von Engagementverbänden}

Wie es Exklusionsverbände für einen geschlossenen Kreis tun, stellen auch legitime Engagementverbände i.e.S. Kollektivgüter bereit, allerdings solche für einen offenen Kreis von Nutzern. Daher besteht zwischen den Nutzenempfängern typischerweise keine Rivalität. Da die Leistungen aber für andere erbracht werden sollen, kann das Exklusionsprinzip bei den Engagementverbänden nicht als Instrument zur Sicherstellung der Finanzierung eingesetzt werden. ${ }^{5}$ Die Finanzierung von Engagementverbänden unterscheidet sich von der von Exklusionsverbänden wegen des Fehlens von Exklusionsmöglichkeiten dadurch, dass Geldspenden, staatliche Zuschüsse und Ausgabenersparnisse in Form von Sachspenden und Freiwilligenarbeit eine große Rolle spielen. Weitere Unterschiede zwischen den Engagementverbänden, die für manche Autoren anscheinend den ,eigentlichen“ (meist allerdings sehr schwammig definierten) „Dritten Sektor“ ausmachen, und den Exklusionsverbänden werden sichtbar, wenn man die idealisierten Darstellungen im Kasten 1 und im Kasten 4 miteinander vergleicht.

Im Kasten 4 (S. 16) sieht man auf der Mitgliederseite eine Zweiteilung: Es gibt einen Förderverein mit Dauermitgliedern, die auch Kontrollrechte haben; ${ }^{6}$ daneben gibt es in der Regel zusätzlich auch noch einen Quasiverein von fallweisen Spendern ohne Kontrollrechte. Außerdem - und das ist charakteristisch - stellen die Engagementverbände kein Kollektivgut zur Förderung der Wohlfahrt ihrer Mitglieder im Wege der egoistischen Selbsthilfe bereit, d.h. zu einer Wohlfahrtsförderung gemäß Smith's (1776) „Wealth of Nations“; statt dessen streben sie nach einer altruistischen Fremdhilfe, d.h. einer Wohlfahrtsförderung gemäß Smith’s (1759) „Theory of Moral Sentiments“. Deshalb kann auch das Kongruenzprinzip nicht realisiert werden: Die Nutzenempfänger unterscheiden sich systematisch von den Kostenträgern

5 Engagement-, Exklusions- und Interessenverbände weisen also Gemeinsamkeiten und Unterschiede auf: Gemeinsam ist allen, dass sie als Nicht-Gebietskörperschaften Kollektivgüter bereitstellen. Bei den Exklusions- und Interessenverbände sind dies aber Güter für einen geschlossenen Kreis von Nutzern, bei den Engagementverbänden dagegen solche für einen offenen Kreis. Engagement- und Interessenverbände können nicht exkludieren, Exklusionsverbänden ist dies dagegen möglich.

6 Juristisch nicht ganz korrekt kann eine philanthropische Stiftung im hier verfolgten Sinnzusammenhang wie ein Verein behandelt werden. Zu den rechtlichen Gemeinsamkeiten und Unterschieden zwischen Vereinen und Stiftungen vgl. z.B. Lex (2005); zur umfassenden Beschreibung des Stiftungswesens vgl. Graf Strachwitz/Mercker (2005) 
(Spendern), und die Kontrollrechte liegen faktisch allein bei der Teilgruppe der Dauerspender. Da aber wie bei den Exklusionsverbänden für ein Niedrighalten der Kosten und eine möglichst effiziente Zielerreichung gesorgt werden sollte, kann man organisatorisch zu Ausschüttungsverboten und Beschränkungen für Verwaltungskostenanteile greifen, um eine versteckte Gewinnorientierung und Selbstbedienung des Managements (weitgehend) auszuschalten. Als weiterer Unterschied zu den Exklusionsverbänden kommt hinzu, dass die Verbandsführung vielfach keine (Vor-) Leistungen einkauft, sondern Transfers an Begünstigte zahlt, die ihrerseits Kollektivgüter für die Öffentlichkeit bereitstellen oder die von der Öffentlichkeit als förderungswürdig betrachtet werden. Gemäß den „Make or buy“-Kriterien kann eventuell auch eine Eigenproduktion der Kollektivgüter gerechtfertigt sein.

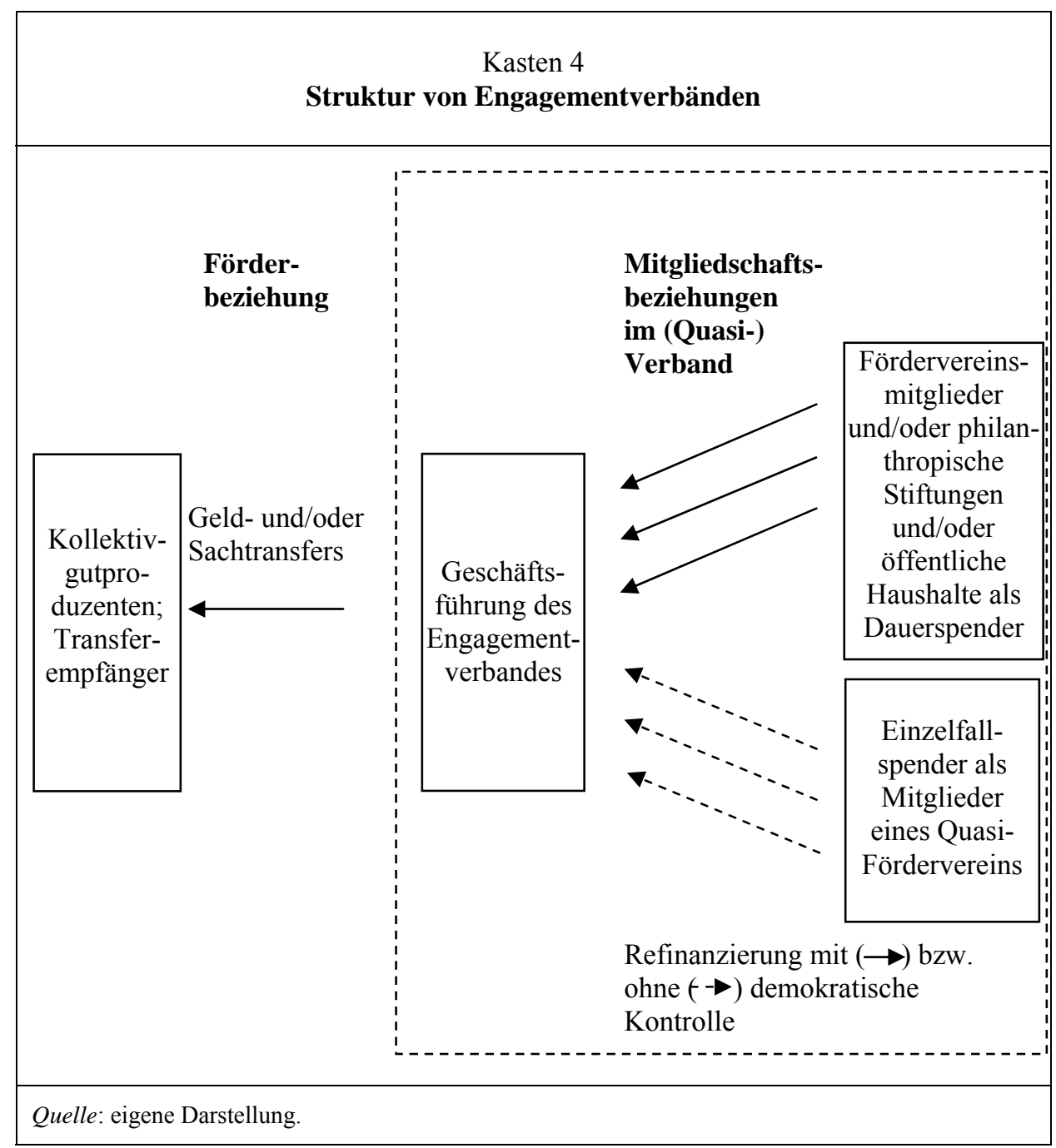


Bei der Gründung eines Engagementverbandes tritt ein Problem auf, das es auch bei Exklusionsverbänden gibt, das insbesondere bei kleineren Engagementverbänden aber in der Regel von ungleich größerer Bedeutung ist: Eine Verbandsgründung für (zahlen- und personenmäßig zunächst unbestimmte) potenzielle Verbandsmitglieder ist als solche ein Kollektivgut, dessen Bereitstellung einen relativ großen Aufwand von Transaktionskosten - hier Gründungskosten - erfordert. Viele Menschen sind deshalb zwar bereit, Beiträge oder Spenden an einen bestehenden Verband zu zahlen, nehmen bei der Übernahme von Gründungslasten aber eine Trittbrettfahrerhaltung ein. Viele wollen darüber hinaus überhaupt nicht Mitglied in einem Verband werden, sondern nur für bestimmte Aktionen - etwa eine Katastrophenhilfe - spenden. Zur Gründung von Engagementverbänden bedarf es deshalb vielfach eines ,sozialen Unternehmers“, der als Mäzen auftritt. Da das Gründungsproblem bei allen Arten von Verbänden auftritt, könnte man seine Lösung auch als ein Kollektivgut im Sinne eines Optionsgutes verstehen: Allen Bürgern wird über Gründungshilfen die Möglichkeit verschafft, in Verbände einzutreten oder Verbänden etwas zu spenden, deren Existenz von den Bürgern als sinnvoll erachtet und gewünscht wird.

Die Finanzierungskompetenz eines Engagementverbandes spiegelt sich - anders als die eines Exklusionsverbandes - nicht in verschiedenen Rechtsformen wider. Alle diese Verbände haben vielmehr eine vereinsähnliche Struktur. Deshalb hat das Extensionsniveau grundsätzlich den Wert 1. Anders als bei den Exklusionsverbänden gibt es bei den Engagementverbänden innerhalb der Kategorie „Vereine“ aber eine Rangfolge der Vereinstypen. Diese korrespondiert - wie im Kasten 5 dargestellt - mit der Möglichkeit des Einwerbens legitimer Subventionskategorien. Da alle Engagementverbände das Extensionsniveau Eins aufweisen, wird die interne Rangfolge nach diesen Subventionskategorien durch die Folge A, B, C bezeichnet. Sie zeigt einen steigenden Subventionswert an, der ökonomisch gerechtfertigt werden muss. Die Verbandstypen innerhalb der Kategorie „Vereine“ sind der Engagementverein mit Gütesiegel (Niveau A), der Engagementverein mit Gemeinnützigkeitsprivileg (Niveau B) und der gemeinnützige Engagementverein mit Zuschussprivileg (Niveau C). Beispiele hierfür werden im Abschnitt 3.2 besprochen.

Wie bereits angedeutet, stehen zur Auswahl des legitimen Extensionsniveaus von Engagementverbänden weder die optimale Bepreisung der Nutzung eines Verbandsguts noch das Kongruenzprinzip als Auswahlkriterien zur Verfügung. Da es um eine Förderung anderer geht, kann kein Nutzungstarif erhoben werden. Außerdem können Zahler und Nutzer nicht identisch sein, denn es stehen ja nur Spenden im weitesten Sinn und staatliche Zuschüsse als Finanzierungsmittel zur Verfügung. Das Subsidiaritätsprinzip kann und sollte aber auch im Bereich der Engagementverbände eingehalten werden, und zwar zum einen mit Blick auf die Konsensfindungskosten und zum anderen mit Blick auf die staatlichen Zuschüsse: Erstere sollten möglichst niedrig sein; letztere sollten auf Hilfen zur Deckung von Transaktionskosten der Gründung und zur Aufrechterhaltung eines minimalen organisatorischen Kerns be- 
schränkt werden und auf die Abgeltung der kostenminimalen Bereitstellung von Kollektivgütern für einen der Zahl nach prinzipiell unbeschränkten Kreis von Nutzern. Außerdem sollte die Bereitstellung so beschaffen sein, dass es auch legitim wäre, zu einer Zwangsfinanzierung durch den Staat zu greifen, dass über die private Bereitstellung also eine Staatsentlastung eintritt.

\begin{tabular}{|c|c|c|c|}
\hline \multicolumn{4}{|c|}{$\begin{array}{l}\text { Kasten } 5 \\
\text { Das Extensionsniveau e von Engagementverbänden }\end{array}$} \\
\hline $\begin{array}{c}\text { E-WERT } \\
\text { (SUKZESSIV- } \\
\text { KATEGORIEN) }\end{array}$ & BEZEICHNUNG & $\begin{array}{l}\text { FINANZIERUNGS- } \\
\text { KOMPETENZ UND } \\
\text { SUBVENTIONS- } \\
\text { KATEGORIE }\end{array}$ & SUBVENTIONSWERT \\
\hline 1.A & $\begin{array}{l}\text { Niveau eines } \\
\text { Engagementvereins } \\
\text { mit Gütesiegel und } \\
\text { (evtl.) Organisati- } \\
\text { onsunterstützung }\end{array}$ & $\begin{array}{l}\text { Spenden und ggf. } \\
\text { Erträge des Stif- } \\
\text { tungskapitals; evtl. } \\
\text { Organisationsun- } \\
\text { terstützung }\end{array}$ & $\begin{array}{l}\text { Wert der Organisati- } \\
\text { onsunterstützung }\end{array}$ \\
\hline 1.B & $\begin{array}{l}\text { Niveau eines } \\
\text { Engagementvereins } \\
\text { mit Gemeinützig- } \\
\text { keitsprivileg }\end{array}$ & $\begin{array}{l}\text { wie bei e }=1 . \text { A } \\
\text { plus steuerliche } \\
\text { Absetzbarkeit von } \\
\text { Spenden }\end{array}$ & $\begin{array}{l}\text { ggf. wie bei e }=1 . \mathrm{A} \\
\text { plus Wert der Steu- } \\
\text { ersubvention }\end{array}$ \\
\hline 1.C & $\begin{array}{l}\text { Niveau eines ge- } \\
\text { meinnützigen } \\
\text { Engagementvereins } \\
\text { mit Zuschuss- } \\
\text { privileg }\end{array}$ & $\begin{array}{l}\text { wie bei } \mathrm{e}=1 . \mathrm{B} \\
\text { plus Finanzsubven- } \\
\text { tion }\end{array}$ & $\begin{array}{l}\text { wie bei e }=1 . \mathrm{B} \text { plus } \\
\text { Wert der Finanzsub- } \\
\text { vention }\end{array}$ \\
\hline & & & \\
\hline
\end{tabular}




\subsection{Beispiele für legitime Engagementverbände im „Dritten Sektor“}

\section{(1) Verein zur Unterstützung bemitleidenswerter Menschen}

Es gibt Vereine, die z.B. schwerkranken Kindern eine Freude machen oder Ärzten in Entwicklungsländern mit Geld- und Sachspenden bei ihrer Tätigkeit helfen wollen. Solche Vereine vermitteln Spendern eine Verwendung von Einkommensteilen zu philanthropischen Zwecken; sie dienen aber nicht der Bereitstellung eines Kollektivgutes, denn es geht hier um die Gestaltung der Eigentumsverwendung der Spender zur Förderung eines von ihnen ausgesuchten Kreises von Hilfeempfängern. „Geholfen“ wird hier zwei abgeschlossenen Kreisen von Nutzenempfängern: den Spendern (die individuelles Einkommen und/oder Vermögen - aus welchen Gründen auch immer - philanthropisch verwenden möchten) und den zur Förderung Ausgesuchten, deren individuelle Wohlfahrt erhöht wird und die mit nicht Ausgesuchten rivalisieren. Mangels Bereitstellung eines Kollektivgutes gehören Unterstützungsvereine nicht zu den Engagementverbänden i.e.S. Anders als bei diesen tritt auch keine Staatsentlastung ein, denn das Kollektivgut „Versicherung gegen soziale Notlagen“ erfüllt der Staat auch ohne die Existenz philanthrophischer Spenden und Zuweisungen dieser Art werden auch nicht auf die staatlichen Leistungen angerechnet. Weil aber zweifellos auf der Spenderseite ein von den meisten Bürgern für besonders moralisch und förderungswürdig angesehenes Engagement vorliegt - wenn auch keines, das der Bereitstellung eines Kollektivgutes dient - und außerdem gleich zu schildernde Verknüpfungen mit Kollektivgütern bestehen, sollen Unterstützungsvereine als Engagementverbände i.w.S. bezeichnet werden.

Solche Vereine leiden unter einem Organisationsproblem, das nach einem - vermutlich weit verbreiteten - Werturteil mit staatlicher Hilfe gelöst werden sollte: Die Gründung solch eines Vereins stellt - wie dargelegt - als solche ein Kollektivgut dar. Potenzielle Spender wissen noch dazu vielfach nicht, ob ein Verein vertrauenswürdig ist, und es ist für engagierte Bürger häufig auch schwierig, einen Verein zu finden, der Hilfe in gerade den Fällen leistet, bei denen man sich selbst engagieren möchte. Die Behebung dieser Informationsmängel kann als Bereitstellung eines Kollektivgutes angesehen werden, welches der Staat gewährleisten sollte. Für kleine Unterstützungsvereine stellen Organisationsprobleme wie die Anmietung eines Raumes für Vereinsversammlungen und die Erledigung rechtlich notwendiger Formalien darüber hinaus Transaktionskosten dar, die hohen Hürden gleichen. Diese Probleme können durch eine Akkreditierung mit Verleihung eines Gütesiegels und dadurch gelöst werden, dass eine Kommune einen Raum für abendliche Vereinsversammlungen in einem Dienstgebäude zur Verfügung stellt und Hilfen bei der Erle- 
digung von Formalitäten sowie in Form einer Kontaktbörse leistet (,Zentren für ein bürgerschaftliches Engagement"). ${ }^{7}$ Mehr wäre nach dem Subsidiaritätsprinzip bei solchen Verbänden nicht angemessen. Das richtige Extensionsniveau wäre somit das eines akkreditierten Engagementvereins mit Gütesiegel und erforderlichenfalls Organisationshilfe $(\mathrm{e}=1 . \mathrm{A})$.

\section{(2) Heimatverein; Denkmalschutzverein}

Vereine, die es sich zur Aufgabe gemacht haben, das Traditionsgut in einer Region oder die Erhaltung von Denkmälern in Deutschland zu fördern, stellen ein Kollektivgut bereit, das auch den Mitgliedern künftiger Generationen dient und bei dessen Nutzung normalerweise keine Rivalität besteht. Sie sollten nicht - auch nicht zum Teil - durch Gebühren finanziert werden, weil dies Nutzer verdrängen würde, obwohl keine Grenzkosten verursacht werden. Das bedeutet, dass die ordnungspolitischen Voraussetzungen für eine Finanzierung über freiwillige Beiträge und eine staatliche Kofinanzierung in einem politisch zu bestimmenden Ausmaß gegeben sind und dass eine Spendenfinanzierung eine Staatsentlastung bei der Pflege des kulturellen Erbes darstellt. Der Staat sollte eine steuerliche Absetzbarkeit der Spenden jedenfalls dann anerkennen, wenn und so weit die Spendenfinanzierung aufgrund des damit gekoppelten bürgerlichen Engagements kostengünstiger als eine staatliche Aufgabenverrichtung ist. Das wohlfahrtsökonomisch „richtige“ Niveau der Förderung zu ermitteln, ist nahezu unmöglich. Die steuerliche Abzugsfähigkeit der Spenden kann aber jedenfalls dann als eine pragmatische Annäherung angesehen werden, wenn sich die Spendenerhebungskosten und die Organisationskosten in Grenzen halten und ein Ausschüttungsverbot für eventuelle Überschüsse besteht. Angemessen ist somit ein Engagementverein mit Gemeinnützigkeitsprivileg (e=1.B).

Zu diesem Lösungsvorschlag, der im Prinzip der Gemeinnützigkeitspraxis ähnelt, sind zwei Anmerkungen angebracht:

- Zum einem verlangt das Zweite Gossensche Gesetz, dass der so genannte Grenznutzen des Geldes in allen Verwendungsarten - privaten wie öffentlichen - gleich groß ist. Das bedeutet, dass alle knappen Güter in einem gewissen manchmal allerdings sehr kleinen - Umfang bereitgestellt werden müssen und dass keines bis zur Sättigungsgrenze bereitgestellt werden darf („Nichtsättigungsaxiom in einer Cobb-Douglas-Welt"). Ein Gemeinnützigkeitsprivileg könnte im Prinzip dazu führen, dass im Vergleich zu anderen Kollektivgütern zuviel von einem spendenfinanzierten Gut (hier dem Denkmalschutz) bereitgestellt wird, weil es an einer institutionell organisierten Konkurrenz um knappe Budgetmittel fehlt. Angesichts sonstiger Abweichungen von „idealen“ Organisationsformen dürfte dieser Mangel aber hinnehmbar sein.

7 Die Enquete-Kommission „Zukunft des Bürgerschaftlichen Engagements“ (2002, S. 147-149) nennt solche Zentren „Freiwilligenagenturen“ und gibt einen Überblick über die Entstehungsund Organisationsformen. 
- Zum anderen erhebt sich die Frage, worin die Kostenvorteile eines Engagementverbandes im Vergleich zum Staat bestehen können. Die Antwort lautet: Zum Teil haben ehrenamtliche Tätigkeiten einen Hobbycharakter, was Transformationskosten senkt: Der pensionierte Lehrer, der Vorstand des Heimatvereins ist und sich um das Heimatmuseum kümmert, arbeitet seiner intrinsischen Motivation wegen kostenfrei. Zum anderen erspart dezentrales Tätigwerden häufig auch Transaktionskosten: Unser Lehrer kennt seine Region und ihr Kulturgut und hat deshalb normalerweise geringere Suchkosten als ein Beamter des Kultusministeriums. Die so begründeten Vorteile spendenfinanzierter Verbände können freilich aufgezehrt werden, wenn das Einwerben der Spenden zu kostspielig wird.

(3) Bereitstellung von Katastrophenhilfe im In- und Ausland

Katastrophenhilfe lässt sich zumindest für die Hilfe im Ausland nicht versicherungsmäßig (und damit über einen Exklusionsverband) organisieren. Sie entspricht ausweislich der Spendenbereitschaft in Notfällen aber offensichtlich dem Willen der Bevölkerung und könnte ihr im Bedarfsfall in Form reziproker ausländischer Hilfe auch selbst zu Gute kommen. So gesehen liegt ein Kollektivgut vor, das im Prinzip auch vom Staat bereitgestellt werden könnte.

Technisch benötigt Katastrophenhilfe das Vorhalten von Gerät und ausgebildetem Personal. Man braucht deshalb einen Verein, der über eine Grundfinanzierung verfügt, über die sich ein genossenschaftliches Hilfsunternehmen in Einsatzbereitschaft halten lässt und Spenden im Bedarfsfall sinnvoll eingesetzt werden können. Eine ordnungspolitisch im Prinzip richtige Lösung könnte pragmatisch so ausgestaltet werden, dass der Staat die Grundfinanzierung bereitstellt und sich damit das Engagement von Bürgern erschließt, die spenden oder mitarbeiten wollen. Die Spenden sollten unter den gleichen Umständen absetzbar sein, wie sie am Beispiel des Denkmalschutzvereins erläutert wurden. Ein zweckmäßiges Extensionsniveau wäre deshalb das eines gemeinnützigen Engagementverbandes mit Zuschussprivileg $(\mathrm{e}=1 . \mathrm{C})$. Deutsche Wohlfahrtsverbände wie der Caritasverband oder das Deutsche Rote Kreuz sind nicht nur Katastrophenhilfsvereine, sondern stellen - ökonomisch betrachtet - eine Mischung von mehreren Organisationstypen dar. Diese haben z.T. den Charakter von Exklusions- und z.T. den von Engagementverbänden, teilweise aber auch den von Erwerbsunternehmen (Hüdepohl 1996). An ihrer Organisation wird vielfach Kritik geübt. Manches daran dürfte berechtigt sein; es wird allerdings oft übersehen, dass die dort Beschäftigten zu einem nicht unerheblichen Teil ehrenamtlich arbeiten und sich deshalb nicht in ein Schema pressen lassen wollen, wie es die Betriebswirtschaftslehre für Beschäftigte in Erwerbsunternehmen fordern würde. Stattdessen verlangen sie ein gewisses Maß an Selbstorganisation, Stressfreiheit und Muße zum kameradschaftlichen Miteinander. Gleichwohl: Ein positiver Subventionswert wäre nur gerechtfertigt, wenn der Staat die Hilfe selbst nicht kostengünstiger bereitstellen könnte. 
Damit ist gezeigt, dass die Tätigkeit der Engagementverbände im „Dritten Sektor“ analog zu jener der Exklusionsverbände jedenfalls dann als ökonomisch gerechtfertigt angesehen werden kann, wenn ein legitimes Extensionsniveau realisiert, d.h. das Subsidiaritätsprinzip bei der Subventionierung ebenso beachtet wird wie die Forderung nach der Eingrenzung von Missbrauchsmöglichkeiten und des Subventionswerts. 


\section{Fazit und Ausblick}

Insgesamt hat die Untersuchung ergeben, dass ein Vereine, Zwangsvereine und (Zwangs-) Genossenschaften umfassender „Dritter Sektor“ aus ordnungsökonomischer Sicht sinnvoll strukturiert werden kann. Die legitimen Verbände in diesem Sektor befassen sich mit der privaten (oder besser: nicht gebietskörperschaftlichen) Bereitstellung unterschiedlicher Arten von Kollektivgütern. Sie verfolgen damit eine Aufgabe, die nicht bloß eine Hilfskonstruktion zum Ausbügeln von Staatsversagen darstellt. Eine ordnungsökonomische Analyse führt somit nicht zu der Aussage, der „Dritte Sektor“ erfülle ökonomisch bloß eine Lückenbüßerfunktion, einer Aussage, wie sie z.B. von Salamon/Anheier (1997, S. 217, und 1998, S. 19) in Anlehnung an Weisbrod (1977) vertreten wird. Man wird allerdings zugeben müssen, dass die Struktur des „Dritten Sektors“ in der Realität international unterschiedliche Abweichungen von der in diesem Aufsatz beschriebenen legitimen Struktur hat. Dies dürfte sich unter anderem auf die folgenden Gründe zurückführen lassen: ${ }^{8}$

- Die Wahl des legitimen Extensionsniveaus ist in der Praxis alles andere als garantiert, denn es gibt für Politiker Anreize, über das Zahlen von illegitimen (wenn auch legalen) Zuschüssen in der gleichen Weise Herrschaftssicherungspolitik zu betreiben, wie man dies bei der Entwicklung der Sozialpolitik beobachten kann (Grossekettler 2004).

- Der „Dritte Sektor“ hat in historischer Perspektive oft auch Aufgaben übernommen, die gar nicht in den Bereich seiner hier abgeleiteten legitimen Tätigkeiten fallen, sondern an sich vom Staat hätten ausgeübt werden müssen. Dies war der Tatsache geschuldet, dass der vormoderne Staat viele seiner eigentlichen Aufgaben vernachlässigt hat. Organisatorische Trägheit einerseits und politische Rücksichtnahme auf die Existenz von überkommenen Organisationen andererseits dürften deshalb im internationalen Vergleich zu sehr unterschiedlichen Grenzziehungen zwischen den Aufgaben des Staates und denen des „Dritten Sektors" geführt haben.

- Verbände im „Dritten Sektor“ haben zur Aufbesserung ihrer Finanzen und zur Verstärkung der Mitgliederbindung oft zu dem Mittel gegriffen, Individualgüter zu besonders günstigen Konditionen anzubieten. Auch dies hat dann zu internationalen Differenzen in der Struktur des „Dritten Sektors“ geführt.

Auf eine wichtige These wurde in diesem Beitrag bisher nicht eingegangen: die These, dass der „Dritte Sektor“ den Nährboden für das Herausbilden gemeinsamer Werte und von gegenseitigem Vertrauen bilde und damit förderlich für Gemeinsinn und demokratisches Miteinander sei, welches als Sozialkapital zur Senkung von

$8 \mathrm{Zu}$ weiteren Erklärungsversuchen für internationale Differenzen vgl. Salamon/Anheier (1997), die sich allerdings auf z.T. anders abgegrenzten Nonprofit-Organisationen beziehen. 
Transaktionskosten und darüber zu einer Erhöhung des Wirtschaftswachstums führe. Diese insbesondere von Putnam $(1993 ; 2001 ; 2002)$ vertretene Hypothese ist plausibel, und es gibt anekdotische Evidenz für ihre Richtigkeit. Sie steht aber in einem gewissen Gegensatz zur These Olsons $(1985 ; 1996)$ von der durch Interessengruppen verursachten Sklerose und der dadurch bewirkten Verminderung des Wirtschaftswachstums, die ebenfalls Plausibilität und anekdotische Evidenz für sich in Anspruch nehmen kann. In diesem Gegensatz spiegelt sich eine Ambivalenz des Sozialkapitalbegriffs, die besonders deutlich wird, wenn man Sozialkapital mit Offe/Fuchs (2001, S. 470) und in Anlehnung an Heiner (1990) als Summe aller Verhaltensdispositionen von Bürgern definiert, die Beiträge zur Senkung von Transaktionskosten in Wirtschaft und Politik leisten. ${ }^{9}$ Transaktionskostensenkungen können nämlich ökonomisch erwünschte Prozesse wie etwa eine verstärkte Arbeitsteilung einem Schmiermittel gleich erleichtern; sie können aber auch Reibungswiderstände abbauen, die sonst als Hürden gegen eine Bildung von heimlichen Kartellen und auf Wettbewerbsbeschränkungen zielenden Interessengruppen sowie (versteckte) Protektionsformen gewirkt hätten. Man wird also wohl versuchen müssen - das hat im Prinzip schon Putnam (1995, S. 76) so gesehen und wird auch in der neueren Literatur betont (Kern 2004) -, zwischen wachstumsfördernden und wachstumshemmenden Gruppen oder Netzwerken und von ihnen vermittelten Einstellungen zu unterscheiden und entsprechende Thesen systematisch empirisch zu testen. Erste Ergebnisse von Analysen, die über Fallstudien hinausgehen, liegen bereits vor. So hat Oelgart (2006) ähnlich wie schon Mill (1948, S. 135 f.) in seinen „Principles“ auf die produktive Funktion informeller Regeln abgestellt und nach wachstumsfördernden Einstellungen in Gruppen gesucht. Er hat mittels einer Faktorenanalyse gezeigt, dass letztlich die Bereitschaft zur Eigenverantwortung und - für manche Kreise ganz „unkorrekt" - Sekundärtugenden solche wachstumsfördernden Einstellungen darstellen (ebenda S. 187). Sozialkapital könnte aus ökonomischer Sicht damit als ein „Set von marktwirtschaftlichen Einstellungen“ definiert werden (ebenda, S.188). Sollten zukünftige Untersuchungen zeigen, dass legitime Exklusions- und/oder Engagementverbände, wie sie hier behandelt wurden, im Gegensatz zum Gros aller politischen Interessengruppen wachstumsförderliche Einstellungen generieren - Vertreter der Historischen Schule hätten vom Erlernen einer marktwirtschaftlichen Wirtschaftsgesinnung gesprochen -, müssten die Wachstumstheorie und manche Teile der Wirtschafts- und Finanzpolitik im Lichte dieser Erkenntnisse neu überdacht werden. Damit eröffnete sich dann allerdings ein weites Feld.

9 Dies ist nur eine von vielen Definitionen von „Sozialkapital“. Einen Überblick über die am weitesten verbreiteten (,klassischen“) Definitionen findet man bei Oelgart (2006, S. 7-35). 


\section{Literatur}

Enquete-Kommission „Zukunft des Bürgerschaftlichen Engagements“ (2002). Bürgerschaftliches Engagement: auf dem Weg in eine zukunftsfähige Bürgergesellschaft, Berlin (Bundestagsdrucksache 14/2351).

Gelbhaar, Siegfried (1998). Ökonomik der Parafiski. Stand und Perspektiven, in: WiStWirtschaftswissenschaftliches Studium, 27. Jg., S. 573-579.

Graf Strachwitz, Rupert, und Florian Mercker (Hrsg., 2005). Stiftungen in Theorie, Recht und Praxis, Berlin.

Grossekettler, Heinz (1984).Ordnungspolitisch legitimierte Genossenschaftsaufgaben. In: W. Jäger und H. Pauli (Hrsg.). Genossenschaften und Genossenschaftswissenschaft, Wiesbaden, S. 57-94.

Grossekettler, Heinz (2000). Steuerstaat versus Gebührenstaat: Vor- und Nachteile. In: U. Sacksofsky und J. Wieland (Hrsg.). Vom Steuerstaat zum Gebührenstaat, Baden-Baden, S. 24-45.

Grossekettler, Heinz (2003). Öffentliche Finanzen. In: D. Bender et al. (Hrsg.). Vahlens Kompendium der Wirtschaftstheorie und Wirtschaftspolitik, München, S. 561-717.

Grossekettler, Heinz (2004). Social Insurance. In: J. B. Backhaus und R. E. Wagner (Hrsg.). Handbook of Public Finance, Boston u.a.O., S. 323-383.

Haibach, Marita (1998). Spezifika der Finanzierung des Dritten Sektors. In: R. Graf Strachwitz (Hrsg.). Dritter Sektor - Dritte Kraft. Versuch einer Standortbestimmung. Stuttgart u.a.O., S. 475-490.

Heiner, Ronald A. (1990). Imperfect Choice and the Origin of Institutional Rules, in: Journal of Institutional and Theoretical Economics (JITE), 146, S. 720-726.

Hüdepohl, Astrid (1996). Organisationen der Wohlfahrtspflege. Eine ökonomische Analyse ausgewählter nationaler und internationaler Institutionen, Berlin.

IHK Hamburg (Hrsg., 2004). Business Improvement District. Quartiersentwicklung durch Eigen(tümer)initiative, Hamburg (Bearbeiter: B. Reichhardt, Th. Koletschka und J. Märkt).

Kern, Kristine (2004). Sozialkapital, Netzwerke und Demokratie. In: A. Klein et al. (Hrsg.). Zivilgesellschaft und Sozialkapital - Herausforderungen politischer und sozialer Integration, Wiesbaden, S. 109-128. 
Kraus, Margit, und Dan Stegarescu (2005). Non-Profit-Organisationen in Deutschland. Ansatzpunkte für eine Reform des Wohlfahrtsstaats. Mannheim (ZEW Dokumentation 05-02).

Lepelmeier, Dirk (1984). Intermediäre Finanzwirtschaften (Parafisci), in: WiSt-Wirtschaftswissenschaftliches Studium. 13. Jg., S. 554-560.

Lex, Peter (2005). Die Grundzüge des Stiftungsrechts. In: R. Graf Strachwitz und F. Mercker (Hrsg.). Stiftungen in Theorie, Recht und Praxis, Berlin, S. 205-209.

Mändle, Eduard (1992). Raiffeisengrundsätze. In: E. Mändle und W. Swoboda (Hrsg.). Genossenschaftslexikon, Wiesbaden, S. 537-539.

Märkt, Jörg (2003). Preise als Steuern. Zur Notwendigkeit einer Besteuerung ohne Trittbrettfahrer angesichts des Steuerwettbewerbs, Freiburg.

Mill, John Stuart (1948). Principles of Political Economy, London.

Oelgart, Niels (2006). Sozialkapital, Kultur und ihr Einfluss auf die wirtschaftliche Entwicklung, Hamburg.

Offe, Claus, und Susanne Fuchs (2001). Schwund des Sozialkapitals? Der Fall Deutschland. In: R Putnam (2001). Gesellschaft und Gemeinsinn. Sozialkapital im internationalen Vergleich, Gütersloh, S. 417-514.

Olson, Mancur Jr. (1968). Die Logik des kollektiven Handelns, Kollektivgüter und die Theorie der Gruppen, Tübingen.

Olson, Mancur Jr. (1985). Aufstieg und Niedergang von Nationen. Ökonomisches Wachstum, Stagflation und Starrheit. Tübingen.

Olson, Mancur Jr. (1996). Big Bills left on the Sidewalk: Why Some Nations are Rich, and Others Poor. In: Journal of Economic Perspectives, Vol. 10, S. 3-24.

Putnam, Robert D. (1993). Making Democracy Work. Civil traditions in modern Italy, Princeton.

Putnam, Robert D. (1995). Bowling alone: America's declining social capital. In: Journal of Democracy, Vol. 6, S. 65-78.

Putnam, Robert D. (Hrsg., 2001). Gesellschaft und Gemeinsinn. Sozialkapital im internationalen Vergleich, Gütersloh.

Putnam, Robert D. (Hrsg., 2002). Democracies in Flux. The Evolution of Social Capital in Contemporary Society, Oxford. 
Salamon, Lester M., und Helmut K. Anheier (1997). Der Nonprofit-Sektor: Ein theoretischer Versuch. In: K.H. Anheier et al. (Hrsg.). Der Dritte Sektor in Deutschland, Berlin, S. 211-246.

Salamon, Lester M., und Helmut K. Anheier (1998). Dritter Sektor und Zivilgesellschaft - Globale Entwicklungen. In: R. Graf Strachwitz (Hrsg.). Dritter Sektor - Dritte Kraft. Versuch einer Standortbestimmung, Stuttgart u.a.O., S. 13-22.

Schmidt-Trenz, Hans-Jörg (1996). Die Logik kollektiven Handelns bei Delegation, Tübingen.

Schmidt-Trenz, Hans-Jörg (2000). Der Staat als Konkurrenz der Selbstverwaltung. In: R. Stober und H. Vogel (Hrsg.). Wirtschaftliche Betätigung der öffentlichen Hand, Köln u.a.O., S. 13-22.

Smith, Adam (1759). The Theory of Moral Sentiments, London.

Smith, Adams (1776). Inquiry into the Nature and Causes of the Wealth of Nations, London.

Weisbrod, Burton A. (1977). The Voluntary Nonprofit-Sektor, Lexington, M.A.

Zimmermann, Horst, und Mathias Krenzer (2001). "Intermediäre” Institutionen in der Perspektive neuer ökonomischer Theorieansätze. WiSt-Wirtschaftswissenschaftliches Studium. 30. Jg., S. $265-272$ 
